\title{
ORIGINAL RESEARCH \\ Multimodal Imaging Does Not Delay Intravenous Thrombolytic Therapy in Acute Stroke
}

\author{
K.M. Salottolo \\ C.V. Fanale \\ K.A. Leonard \\ D.F. Frei \\ D. Bar-Or
}

BACKGROUND AND PURPOSE: Patients with acute ischemic stroke require immediate medical treatment, and a CT to rule out hemorrhage is required before tPA. We adapted our protocol to include multimodal CT: unenhanced CT, CTA, and PCT. The purpose of this study was to determine whether multimodal CT imaging delays initiation of IV tPA beyond 60 minutes from hospital arrival.

\begin{abstract}
MATERIALS AND METHODS: All patients admitted during 3 years through the ED with a stroke alert and time from symptom onset to hospital arrival $<2.5$ hours were included. We examined 2 subgroups (multimodal CT versus unenhanced CT) to determine whether multimodal CT delayed IPA administration. Logistic regression was used to identify variables that predicted tPA within 60 minutes.
\end{abstract}

RESULTS: There were 123 patients in the analysis, including 108 patients who were examined with multimodal CT. The median time from arrival to tPA was 56 minutes and was shorter for patients examined with multimodal CT (55 versus 78 minutes, $P=.02$ ). After adjustment, variables that were associated with tPA administration within 60 minutes included prehospital stroke alert (OR $=3.47, P=$ $.03)$, time to $\mathrm{CT}(\mathrm{OR}=0.94, P=.01)$, and onset-to-arrival time (OR $=1.02, P=.04)$. There was no statistically significant difference in the odds of receiving timely tPA for multimodal versus unenhanced CT $(\mathrm{OR}=3.99, P=.07)$.

CONCLUSIONS: In our single-center experience, the use of multimodal imaging in patients with acute stroke did not delay IV tPA beyond 60 minutes. Further study is needed to assess the feasibility of the routine use of multimodal imaging in the acute stroke setting.
$\mathbf{P}$ atients with an acute ischemic stroke require immediate medical treatment, and administration of a thrombolytic agent is the paramount consideration. Recombinant $\mathrm{PA}$ is the only currently proved treatment for ischemic stroke and has been shown to improve long-term functional dependence. ${ }^{1}$ The current recommended time to treatment with IV tPA is 3 hours, while there is no evidence-based time window for IA tPA at this time. A CT scan to rule out hemorrhage or infarct is required before thrombolytic therapy, but the addition of an intra- and extracranial vascular study (CTA) as well as a brain perfusion study (PCT) may be of benefit to help clinicians determine whether a patient is a candidate for IA therapy. ${ }^{2,3}$

The rapid time to initiate treatment from symptom onset is the greatest barrier to wider application of thrombolytic therapy, but both prehospital and in-hospital delays exclude eligible patients from receiving IV tPA. While prehospital delays have declined significantly from 1981 to 2007, no appreciable

Received July 1, 2010; accepted after revision September 23.

From the Trauma Research Department (K.M.S., D.B.-0.) and the Stroke Center (C.V.F., K.A.L., D.F.F.), Swedish Medical Center, Englewood, Colorado; Trauma Research Department (K.M.S., D.B.-O.), St. Anthony Central Hospital, Denver, Colorado; Radiology Imaging Associates (D.F.F.), Englewood, Colorado; and Colorado Neurological Institute (C.V.F., D.F.F.), Englewood, Colorado.

Paper previously presented at: Annual Meeting of the American Society of Neuroradiology, May 15-20, 2010; Boston, Massachusetts.

This work was supported by the Swedish Medical Center, Englewood, Colorado.

Please address correspondence to David Bar-Or, MD, Trauma Research Department, Swedish Medical Center, 501 E Hampden Ave, Room 4-454, Englewood C0, 80113; e-mail: dbaror@dmibio.com

DOI 10.3174/ajnr.A2394 changes for in-hospital delay times were observed during the same period. ${ }^{4}$ A recent systematic review reported that inhospital times are consistently longer than the recommended NINDS goals. ${ }^{5}$ These goals, published in 1996, recommend the following: 10 minutes from door to ED evaluation, 15 minutes from door to neurologist notification, 25 minutes from door to initiation of CT scan, 45 minutes from door to CT interpretation, and 60 minutes from door to tPA administration. $^{6}$

We adapted our acute stroke protocol in 2003 to include a multimodal CT scan before IV tPA use. The protocol includes unenhanced CT, PCT, and CTA. Since then, the American Heart Association/American Stroke Association recommended, in 2007, that emergency treatment of stroke not be delayed to obtain multimodal imaging studies despite their clinical utility. ${ }^{7}$ The purpose of this study was to determine whether multimodal CT imaging delays the initiation of tPA beyond a goal of 60 minutes from hospital arrival.

\section{Materials and Methods}

\section{Study Design, Setting, and Population}

We conducted a retrospective cohort study of patients with stroke seen at SMC Stroke Center, located in the south Denver metropoliton area. There are 4 attending neurologists and 8 neuroradiologists who cover stroke alert at SMC and 34 EMS agencies within the greater metropolitan area that service SMC. Consideration for IV tPA is determined by using the NINDS study protocol. All patients admitted through the ED with a stroke alert and time from symptom onset to 


\begin{tabular}{|c|c|c|c|}
\hline Characteristic & $\begin{array}{c}\text { Multimodal Imaging } \\
(n=108)\end{array}$ & $\begin{array}{c}\text { Unenhanced CT Alone } \\
(n=15)\end{array}$ & $P$ Value \\
\hline Age $\geq 65$ & $65.7(71)$ & $80.0(12)$ & 0.38 \\
\hline Female sex & $59.3(64)$ & $60.0(9)$ & 0.96 \\
\hline Prehospital stroke notification & $83.3(90)$ & $73.3(11)$ & 0.47 \\
\hline Peak arrival time (12 PM to $12 \mathrm{AM}$ ) & $39.8(43)$ & $40.0(6)$ & 0.99 \\
\hline Weekday arrival (Monday-Friday) & $70.4(76)$ & $66.7(10)$ & 0.77 \\
\hline Initial NIHSS Score $\geq 8$ & $63.8(67)$ & $46.7(7)$ & 0.20 \\
\hline Onset-to-arrival time ${ }^{\mathrm{a}}$ & $48(35-75)$ & $50(30-70)$ & 0.99 \\
\hline Arrival to CT start time ${ }^{a}$ & $11(7-17)$ & $15(10-30)$ & 0.11 \\
\hline Door-to-needle time ${ }^{a}$ & $55(41-75)$ & $78(53-114)$ & 0.02 \\
\hline Year $^{b}$ & & & 0.56 \\
\hline 2006 & $30.6(33)$ & $40.0(6)$ & \\
\hline 2007 & $32.4(35)$ & $26.7(4)$ & \\
\hline 2008 & $37.0(40)$ & $33.3(5)$ & \\
\hline
\end{tabular}

Event times are shown as median (IQR)

${ }^{b}$ Cochran-Armitage test for trend.

hospital arrival $<2.5$ hours during a 3-year period were included (January 2006 to December 2008). Patient exclusions were as follows: treatment with IV tPA at the referring facility, treatment exclusively with IA tPA, and a contraindication to IV tPA.

The analysis consisted of 2 patient subgroups: 1) patients who were examined by using multimodal CT, and 2) patients examined with unenhanced CT of the head. Patients who were not clinically suspected on the basis of presentation to be candidates for IA tPA (small vessel stroke or too mild) were not examined with multimodal CT. Multimodal CT examinations were performed as follows: unenhanced CT, PCT, and then CTA.

CT examinations were performed on either a LightSpeed 16-section scanner (GE Healthcare, Buckinghamshire, United Kingdom) or a Somatom Sensation 64-section scanner (Siemens, Erlangen, Germany) beginning in March 2008. Examinations were available 24/7 with in-house technologists. Non-contrast CT parameters included the following: 3-mm section thickness, $310 \mathrm{~mA}$, and $120 \mathrm{kV}$ (peak) (Siemens 64-section) or a 2.5-mm section thickness through the posterior fossa and a $5-\mathrm{mm}$ section thickness through the top of the head, Smart $\mathrm{mA}$, and $120 \mathrm{kVp}$ (GE 16-section). PCT of the head scan parameters included the following: $40 \mathrm{~mL}$ of nonionic contrast agent (iopamidol, Isovue 370; Bristol-Myers Squibb, Princeton, New Jersey), $160 \mathrm{~mA}$ with 1-second rotation time, $80 \mathrm{kVp}$ for 60 seconds with a 4 -second delay, typical injection rate of $4 \mathrm{~mL} / \mathrm{s}, 9.6-\mathrm{mm}$ section thickness for 28.8-mm coverage (Siemens 64-section) or a 5-mm section thickness for $20 \mathrm{~mm}$ of coverage (GE 16-section). CTA of the head and neck parameters included the following: $70 \mathrm{~mL}$ of nonionic contrast agent (Isovue 370), $120 \mathrm{kVp}$, and CARE Dose 4D (Siemens) with 0.5 -second rotation time and 1-mm section thickness (Siemens 64-section) or $120 \mathrm{kVp}$ and Smart $\mathrm{mA}$ with a 0.6 -section rotation time and 1.25-mm section thickness (GE 16-section).

\section{Outcomes and Covariates}

The primary outcome of the study was whether time from hospital arrival to administration of IV tPA (door-to-needle time) was within the NINDS and SMC goal of 60 minutes $(\leq 60$ minutes versus $>60$ minutes) and whether this differed by the use of multimodal imaging (ie, the 2 subgroups). Secondarily, we sought to determine independent predictors of IV tPA initiation within 60 minutes.

We examined the following categoric variables: age ( $<65$ versus $\geq 65$ ), sex, initial NIHSS scores ( $0-7$ versus $\geq 8$ ), prehospital notification by EMS/transferring facility (versus ED stroke alert), peak ar- rival time (12:00 PM to 11:59 PM versus 12:00 AM and 11:59 AM), weekday arrival (Monday through Friday versus weekend), and year (2006 versus 2007 versus 2008). Time from onset to arrival and time from arrival to CT were examined continuously. We chose to examine these covariates because they were available and complete for patients across the 3-year study period and because we thought that they could potentially influence the door-to-needle time and the decision to use multimodal CT. Because the timing of events was not normally distributed, all events (onset to arrival, arrival to CT, arrival to tPA) are reported in minutes as a median (IQR).

\section{Statistical Analyses}

Statistical analyses were performed by using SAS software, Version 9.1.3 (SAS Institute, Cary, North Carolina). Wilcoxon rank sum tests were used to determine whether the door-to-needle time differed with multimodal imaging. $\chi^{2}$ tests (the Pearson test, the Fisher exact test, and the Cochran-Armitage test for trend) were used to determine clinical/demographic differences by multimodal CT subgroup (Table 1). $\chi^{2}$ tests were also used to determine clinical/demographic differences in patients who met the 60-minute goal of IV tPA initiation versus those who did not (Table 2).

Logistic regression analysis was used to determine which variables independently predicted whether IV tPA was initiated within 60 minutes from arrival (Table 3 ). All variables with a $P$ value $<.20$ based on $\chi^{2}$ univariate tests were adjusted for in the regression model. ORs of $>1.0$ represented an increased odds of receiving IV tPA in 60 minutes. Statistical significance was set at $P<.05$.

\section{Results}

\section{Population Demographics}

There were 422 patients who met inclusion criteria, with 123 patients in the analysis. Primary reasons for exclusion from analysis were a contraindication to $\mathrm{PPA}$, IV tPA received at an outside hospital, IA tPA used without IV tPA, and unavoidable reasons (not initially a stroke alert, family declined consent, unclear examination, recent trauma, stroke missed in the ED). Most patients were female (59.4\%), with a median age of 76 years and a median initial NIHSS score of 11 . The median onset-to-arrival time was 49 minutes (IQR, 35-73 minutes), and the median arrival to CT time was 11 minutes (IQR, 7-19 minutes). 


\begin{tabular}{|c|c|c|c|}
\hline Characteristic & $\begin{array}{l}\leq 60 \text { Minutes to tPA } \\
(n=67)\end{array}$ & $\begin{array}{l}>60 \text { Minutes to tPA } \\
(n=56)\end{array}$ & $P$ Value \\
\hline Multimodal CT imaging & $92.5(62)$ & $82.1(46)$ & .08 \\
\hline Age $\geq 65$ & $68.7(46)$ & $66.1(37)$ & .76 \\
\hline Female sex & $59.7(40)$ & $58.9(33)$ & .93 \\
\hline Prehospital stroke notification & $89.6(60)$ & $73.2(41)$ & $.02^{\mathrm{a}}$ \\
\hline Peak arrival time (12 PM to $12 \mathrm{AM}$ ) & $43.3(29)$ & $35.7(20)$ & .39 \\
\hline Weekday arrival (Monday-Friday) & $67.2(45)$ & $73.2(41)$ & .47 \\
\hline Initial NIHSS score $\geq 8$ & $68.2(45)$ & $53.7(29)$ & .10 \\
\hline Onset-to-arrival time ${ }^{\mathrm{a}}$ & $50(35-83)$ & $44(35-63)$ & .10 \\
\hline Arrival to CT start time ${ }^{\mathrm{a}}$ & $10(6-15)$ & $14(9-26)$ & .01 \\
\hline Door-to-needle time ${ }^{b}$ & $43(37-50)$ & $83.5(70-95)$ & $<.001$ \\
\hline Year $^{b}$ & & & .25 \\
\hline 2006 & $34.3(23)$ & $28.6(16)$ & \\
\hline 2007 & $34.3(23)$ & $28.6(16)$ & \\
\hline 2008 & $31.4(21)$ & $42.9(24)$ & \\
\hline
\end{tabular}

a Event times are shown as median (IQR)

${ }^{\mathrm{b}}$ Cochran-Armitage test for trend.

\begin{tabular}{|c|c|c|c|}
\hline Variable & OR & $95 \% \mathrm{Cl}$ & $P$ Value \\
\hline Multimodal CT & 3.99 & $0.91-17.49$ & .07 \\
\hline Alerted by EMS/transfer & 3.47 & $1.11-10.88$ & .03 \\
\hline NIHSS score $\geq 8$ (vs $0-7$ ) & 1.10 & $0.46-2.63$ & .83 \\
\hline Time from onset (min) & 1.02 & $1.00-1.03$ & .04 \\
\hline Time from admit to CT (min) & 0.94 & $0.90-0.99$ & .01 \\
\hline
\end{tabular}

There were 108 patients who had multimodal CT imaging $(87.8 \%)$ and 15 patients who did not. No demographic or clinical differences were detected by multimodal imaging subgroup (Table 1). Patients were examined with unenhanced CT instead of multimodal CT for multiple various reasons, in particular a deficit too mild for IA consideration $(n=4)$, advanced age $(n=2)$, or an unknown reason $(n=3)$. One patient each had unenhanced CT for the following reasons: not initially recognized as a stroke, stage 4 cancer, MR imaging/MR angiography performed per neurologist request, elevated creatinine level, history of iodine allergy, and allergic reaction during PCT so that CTA was not performed.

Patients whose door-to-needle time was $\leq 60$ minutes were more likely to have had prehospital stroke alert notification (89.6\% versus $73.2 \%, P<.02)$, a longer time from onset to arrival ( $>1$ hour, $40.3 \%$ versus $26.8 \%$ ), and a shorter time from arrival to CT ( $<15$ minutes, $82.1 \%$ versus $60.7 \%$ ) than patients who did not meet the 60 -minute goal (Table 2 ).

\section{Time to IV tPA Administration}

The median door-to-needle time was 56 minutes (IQR, 42-82 minutes). Overall, $54.5 \%(n=67)$ of patients received IV tPA within the 60-minute goal.

The median door-to-needle time was significantly shorter for patients who had multimodal imaging versus those who did not (55 minutes versus 78 minutes, $P=.02$ ). Before adjustment, there was an increased odds of receiving IV tPA within 60 minutes for patients with multimodal imaging; however, this did not reach statistical significance $(\mathrm{OR}=2.70$, $P=.09$ ).

After adjustment, the trend toward timely IV tPA initiation in patients with multimodal imaging continued, with a 4-fold increased odds of meeting the 60 -minute goal for patients who

had a multimodal CT versus those who had an unenhanced $\mathrm{CT}$ alone $(\mathrm{OR}=3.99, P=.07)$. However, we were unable to show a statistically significant difference in the odds of timely tPA for multimodal CT versus unenhanced CT. Significant predictors of IV tPA administration within the 60-minute goal were as follows: prehospital stroke alert notification $(\mathrm{OR}=$ $3.47, P=.03)$, prompt time to CT $(\mathrm{OR}=0.94, P=.01)$, and delayed time from symptom onset to arrival $(\mathrm{OR}=1.02, P=$ .04) (Table 3).

\section{Discussion}

There were 2 principal findings in this study. First, the use of multimodal imaging in patients with acute stroke did not delay administration of IV tPA beyond a goal of 60 minutes from patient arrival. Second, variables that predicted IV tPA use within the 60-minute goal included prehospital stroke alert notification, a longer time from symptom onset to arrival, and a shorter time from arrival to CT examination.

Similar to our findings, prehospital stroke notification has been shown to decrease door-to-needle time and increase the percentage of patients who received IV tPA. ${ }^{8-10} \mathrm{Kim}_{\text {et }} \mathrm{al}^{10}$ implemented a prehospital notification hotline and increased the percentage of patients receiving IV tPA from $6.4 \%$ to $14.3 \%$. Abdullah et $\mathrm{al}^{8}$ increased, from $21 \%$ to $41 \%$, the percentage of patients receiving IV tPA with advanced EMS notification.

Romano et al ${ }^{11}$ previously observed an inverse relationship between arrival and thrombolysis. We also believe the most likely explanation for the inverse relationship we observed is that a sense of urgency was lacking in patients arriving with sufficient time to initiate IV tPA, leading to in-hospital delays. This observation is reflected in the delayed time from CT interpretation to IV tPA for patients with unenhanced CT versus multimodal CT (56 minutes versus 43.5 minutes), though the difference did not reach statistical significance $(P=.07)$.

There are several advantages to performing a multimodal CT scan for diagnosis and treatment management. First, stroke differentiation (infarct versus salvageable ischemic stroke) helps clinicians determine whether a patient is a candidate for IA therapy. Even though IA tPA is not approved by the US Food and Drug Administration to treat acute ischemic 
stroke, there are advantages to arterial-versus-venous administration of tPA, including higher rates of recanalization, potential expansion of the time window out to 6 hours, lower doses of thrombolytic agent used compared with systemic or intravenous $\mathrm{PAA}$, and treatment for patients who were not able to receive IV tPA (bleeding risk, pregnant, other medical contraindication). One-third of patients who were examined with multimodal CT received IV and IA tPA in our study $(n=35)$. CT angiography was used to identify the origin of infarction and the site of cerebral artery occlusion by assessing the intraand extracranial vasculature, while CT perfusion was used to predict which tissue was salvageable with reperfusion and which would die without it. ${ }^{3} \mathrm{CT}$ angiography is more sensitive (70\%) in detecting early irreversible ischemia versus unenhanced CT (40\% sensitivity) and more accurately predicts final infarct volume. ${ }^{2}$ Multimodal CT increases the sensitivity of stroke detection as well as the prediction of the final size of the infarct, compared with unenhanced CT, CTA, and PCT alone. ${ }^{12}$ Moreover, these CT methods can be used immediately after unenhanced CT, they can be used to exclude hemorrhage, imaging time is fast ( $<5$ minutes), and no special hardware is required. ${ }^{13}$

Second, fewer trips to the CT scanner during a patient's hospital stay reduce both transfer-related complications and clinicians' and CT technologists' workloads, increasing efficiency. Transports can impact patients in 2 ways: The actual movement (acceleration, changes in posture) can influence neurologic and physiologic changes, and changes in the environment (noise, surface changes, and equipment changes) can generate added physiologic stress to critically ill patients. ${ }^{14} \mathrm{~A}$ recent observational study revealed 66 adverse events among 290 intrahospital transports of critically ill patients from the ED to the intensive care unit, including some admissions after a CT scan. ${ }^{15}$ The multimodal CT imaging protocol reduces the need for a second trip to the CT scanner, which would otherwise be warranted in critically ill patients to determine candidacy for IA tPA.

Finally, obtaining scans that may be done eventually during the hospital stay has been shown to be cost-effective. In 1000 patients 70-74 years of age with acute stroke, the most costeffective strategy was to scan all patients immediately (reducing costs and increasing independent survival) versus scanning immediately only patients on anticoagulants or having life-threatening conditions, or scanning no patients immediately. ${ }^{16}$

Three primary disadvantages to performing multimodal imaging before the decision for IV tPA exist. First, there is the potential for delayed treatment with IV tPA because the scanning/processing times are shorter for unenhanced CT versus multimodal imaging (approximately 5 minutes versus 15 minutes, respectively), and the sooner IV tPA can be given the greater the benefit. ${ }^{1}$ Our original intent of performing multimodal CT on all patients with an acute stroke alert was to remove the guesswork concerning who should be scanned with CTA/PCT to determine candidacy for IA thrombolytics. Our results show that multimodal imaging neither increased door-to-needle time nor increased the time from CT to IV tPA initiation. We believe that using this standard-of-care protocol may not extend the door-to-needle time because the protocol removes the decision-making step of whether to perform multimodal CT imaging based on various clinical indices (severity of and changes in NIHSS score, specific symptom, and so forth).

Second, performing CT by using a contrast agent without obtaining creatinine levels could lead to a small but significant risk of renal problems. Mehdiratta et $\mathrm{al}^{17}$ reported that $8 \%$ $(n=11)$ of patients would have been ineligible to receive contrast on the basis of elevated creatinine levels. However, 10 of 11 patients had a known history of renal disease. The authors concluded that given the reversibility of contrast-induced nephropathy, the benefits of CTA/PCT outweigh the risks if a proper history is taken. During the study period, our practice was to take a thorough history and perform CTA/PCT before obtaining creatinine results. We recently began using a point-of-service device that allows a creatinine reading within 5 minutes of ED arrival so that multimodal CT is not performed in patients with elevated creatinine levels.

Third, performing multimodal CT involves longer scanning times and the use of nonionic contrast agent, which increases a patient's exposure to radiation. CT examinations in the United States have increased more than 3-fold from 1993 to $2009 .{ }^{18}$ In a previous study performed at SMC, we identified the cumulative effective dose of radiation from head CT and CT of the head and neck following trauma to be 3.86 and 9.04 $\mathrm{mSv}$, respectively. ${ }^{19}$ The Biological Effects of Ionizing Radiation Report VII estimates that 1 in 1000 patients would develop cancer from a CT exposure of $10 \mathrm{mSv} .{ }^{20} \mathrm{MR}$ imaging has also been suggested as an alternative to CT to avoid the risk associated with radiation dose. ${ }^{21}$

Although MR imaging may be diagnostically superior to $\mathrm{CT},{ }^{22-24}$ recent studies reported delays to imaging or IV tPA for MR imaging versus CT. In a study of 195 patients with acute stroke in Korea, the median delay to imaging was 34 minutes with CT versus 69 minutes for MR imaging. ${ }^{25}$ Kang et $\mathrm{al}^{26}$ observed a significant increase in door-to-needle time with MR imaging versus CT (87 minutes versus 68 minutes, respectively); however, discharge modified Rankin Scale scores were not affected by time to treatment or the use of MR imaging. At our stroke center, MR imaging technologists are on call after $10 \mathrm{PM}$; in general, MR imaging is more expensive, time-consuming, and difficult to interpret than CT. For these reasons, we prefer multimodal CT versus multimodal MR imaging.

Limitations to the study exist. Primarily, there were only 15 patients in the group who did not undergo multimodal CT. We did not find any differences in patient and clinical demographics in the multimodal CT subgroup, but this finding may be due to the small sample size in the patients examined with unenhanced CT alone. We do not believe that a selection bias exists; there are some patients whose conditions are not appropriate for multimodal imaging but may still be appropriate for tPA, including patients with small vessel strokes. Even if patients were selected to be examined with unenhanced CT only, this selection should not have resulted in a delay in IV tPA. Patients underwent unenhanced CT because multimodal CT was contraindicated (iodine allergy, elevated creatinine levels) or multimodal CT could increase the potential for harm (contrast agent, superfluous radiation) without providing additional benefit because IA therapy was not a consideration. 
Second, this analysis was conducted in a single high-volume primary stroke center. The results of this study may not be applicable to other centers where interventional neuroradiology is not available. In addition, at our center, the neurologist is in the ED within 5 minutes of stroke alert, is present during the CT examination, and reads and interprets the findings of the CT examination with the radiologist in real-time. Smaller centers and centers with fewer resources may not have the capability to perform a multimodal CT examination in the IV tPA window.

Third, all data were collected retrospectively from 2 data bases: the Get with the Guidelines data base (http:// www.americanheart.org/presenter.jhtml?identifier $=$ 3016522) and our in-house Stroke Tracker data base (http:// www.fileguru.com/apps/free_key_stroke_tracker). There are potentially unmeasured differences in the population, in the multimodal CT imaging subgroup, which we were not able to examine due to the use of pre-existing data. Errors in data entry and data collection are also more common in retrospective analyses than in data that are collected prospectively. Finally, due to data-retrieval limitations, we were unable to examine time to IPA with unenhanced CT before the institution of the multimodal CT protocol (before 2003).

\section{Conclusions}

In our single-center experience, the use of multimodal CT imaging in patients with acute stroke did not delay administration of IV tPA beyond a goal of 60 minutes from patient arrival. Further study is needed to assess the feasibility and safety of the routine use of multimodal CT imaging in the acute stroke setting.

\section{References}

1. Hacke W, Donnan G, Fieschi C, et al. Association of outcome with early stroke treatment: pooled analysis of ATLANTIS, ECASS, and NINDS rt-PA stroke trials. Lancet 2004;363:768-74

2. Camargo EC, Furie KL, Singhal AB, et al. Acute brain infarct: detection and delineation with CT angiographic source images versus nonenhanced CT scans. Radiology 2007;244:541-48

3. Schaefer PW, Roccatagliata L, Ledezma C, et al. First-pass quantitative CT perfusion identifies thresholds for salvageable penumbra in acute stroke patients treated with intra-arterial therapy. AJNR Am J Neuroradiol 2006;27:20-25

4. Evenson KR, Foraker RE, Morris DL, et al. A comprehensive review of prehospital and in-hospital delay times in acute stroke care. Int $J$ Stroke 2009;4:187-99

5. Evenson KR, Rosamond WD, Morris DL. Prehospital and in-hospital delays in acute stroke care. Neuroepidemiology 2001;20:65-76

6. National Institute of Neurological Disorders and Stroke. Proceedings of a National Symposium on Rapid Identification and Treatment of Acute Stroke, December 12-13, 1996, Bethesda, Maryland. NIH Publication No. 97-4239 ed. http:// www.ninds.nih.gov/news_and_events/proceedings/strokeworkshop.htm. Last edited July 01, 1999. Accessed April 29, 2010.
7. Adams HP Jr, del Zoppo G, Alberts MJ, et al. Guidelines for the early management of adults with ischemic stroke: a guideline from the American Heart Association/American Stroke Association Stroke Council, Clinical Cardiology Council, Cardiovascular Radiology and Intervention Council, and the Atherosclerotic Peripheral Vascular Disease and Quality of Care Outcomes in Research Interdisciplinary Working Groups - the American Academy of Neurology affirms the value of this guideline as an educational tool for neurologists. Stroke 2007;38:1655-711

8. Abdullah AR, Smith EE, Biddinger PD, et al. Advance hospital notification by EMS in acute stroke is associated with shorter door-to-computed tomography time and increased likelihood of administration of tissue-plasminogen activator. Prehosp Emerg Care 2008;12:426-31

9. Belvis R, Cocho D, Marti-Fabregas J, et al. Benefits of a prehospital stroke code system: feasibility and efficacy in the first year of clinical practice in Barcelona, Spain. Cerebrovasc Dis 2005;19:96-101

10. Kim SK, Lee SY, Bae HJ, et al. Pre-hospital notification reduced the door-toneedle time for IV t-PA in acute ischaemic stroke. Eur J Neurol 2009;16:1331-35

11. Romano JG, Muller N, Merino JG, et al. In-hospital delays to stroke thrombolysis: paradoxical effect of early arrival. Neurol Res 2007;29:664-66

12. Kloska SP, Nabavi DG, Gaus C, et al. Acute stroke assessment with CT: do we need multimodal evaluation? Radiology 2004;233:79-86

13. Meuli RA. Imaging viable brain tissue with CT scan during acute stroke. Cerebrovasc Dis 2004;17 (suppl 3):28-34

14. Fanara B, Manzon C, Barbot O, et al. Recommendations for the intra-hospital transport of critically ill patients. Crit Care 2010;14:R87. Epub 2010 May 14

15. Gillman L, Leslie G, Williams T, et al. Adverse events experienced while transferring the critically ill patient from the emergency department to the intensive care unit. Emerg Med J 2006;23:858-61

16. Wardlaw JM, Seymour J, Cairns J, et al. Immediate computed tomography scanning of acute stroke is cost-effective and improves quality of life. Stroke 2004;35:2477-83

17. Mehdiratta M, Schlaug G, Kumar S, et al. Reducing the delay in thrombolysis: is it necessary to await the results of renal function tests before computed tomography perfusion and angiography in patients with code stroke? J Stroke Cerebrovasc Dis 2008;17:273-75

18. Berrington de Gonzalez A, Mahesh M, Kim KP, et al. Projected cancer risks from computed tomographic scans performed in the United States in 2007. Arch Intern Med 2009; 169:2071-77

19. Salottolo K, Bar-Or R, Fleishman M, et al. Current utilization and radiation dose from computed tomography in patients with trauma. Crit Care Med 2009;37:1336-40

20. Committee to Assess Health Risks from Exposure to Low Levels of Ionizing Radiation and National Research Council. Health Risks from Exposure to Low Levels of Ionizing Radiation: BEIR VII. Washington, DC: National Research Council; 2006

21. Semelka RC, Armao DM, Elias J Jr, et al. Imaging strategies to reduce the risk of radiation in CT studies, including selective substitution with MRI. J Magn Reson Imaging 2007;25:900-09

22. Chalela JA, Kidwell CS, Nentwich LM, et al. Magnetic resonance imaging and computed tomography in emergency assessment of patients with suspected acute stroke: a prospective comparison. Lancet 2007;369:293-98

23. Fiebach JB, Schellinger PD, Gass A, et al. Stroke magnetic resonance imaging is accurate in hyperacute intracerebral hemorrhage: a multicenter study on the validity of stroke imaging. Stroke 2004;35:502-06

24. Fiebach JB, Schellinger PD, Jansen $O$, et al. CT and diffusion-weighted MR imaging in randomized order: diffusion-weighted imaging results in higher accuracy and lower interrater variability in the diagnosis of hyperacute ischemic stroke. Stroke 2002;33:2206-10

25. Choi JC, Kang SY, Kang JH, et al. Are in-hospital delays important obstacles in thrombolytic therapy following acute ischemic stroke? J Clin Neurol 2007;3:71-78

26. Kang DW, Chalela JA, Dunn W, et al. MRI screening before standard tissue plasminogen activator therapy is feasible and safe. Stroke 2005;36:1939-43 